\title{
Wastewater Monitoring System in Industrial Workshop Based on Wireless Sensor Network
}

\author{
https://doi.org/10.3991/ijoe.v13i03.6860 \\ Minhai Zhang* \\ Hunan Vocational College of Railway Technology, Zhuzhou, China \\ lishijun040482@qq. com \\ Shuangxiang She \\ Powerchina Zhongnan Engineering Co Ltd., Changsha, China \\ $15116380139 @ 126 . \mathrm{com}$
}

\begin{abstract}
In this paper, regarding the complexity of polluted water, through analyzing the water environment and its surrounding factors, a water quality monitoring system architecture based on wireless sensor networks is proposed. Firstly, the water quality parameters collected by the sensor are processed by the signal conditioning circuit and then transmitted to the sensor nodes so as to realize the data acquisition of the water quality parameters. Afterwards, through the function analysis of each node in the ZigBee network, the hardware design and software program design of the network coordinator node and the routing node are completed. After joint debugging of the hardware and software, point-to-point communication and system networking of the ZigBee network are achieved. Finally, the results of experiments show that the system can realize the data acquisition and wireless transmission of the temperature and $\mathrm{PH}$ parameters of different solutions. Furthermore, each node realizes its own function and meets the design requirements of the system.
\end{abstract}

Keywords-wireless sensor network, wastewater monitoring system, ZigBee network, PH parameters

\section{$1 \quad$ Introduction}

In order to fully and accurately understand the current situation of water pollution, as well as achieve early detection of water pollution, provide early warning for downstream water quality safety precautions and realize timely tracking of sources of pollution, we need to grasp the real-time water environment parameters of the monitoring region. While if we are to master and understand water pollution and developmental trends of a river or a region, and to make a realistic assessment of the water quality, a large number of representative monitoring data must be considered. At present, river water pollution in China is serious. Traditional water quality monitoring methods also have many problems, such as a long water quality monitoring cycle, water quality parameters sampling process consuming much manpower and resources, ag- 
ing equipment and instruments, and insufficient data accuracy. The purpose of this paper is to adopt advanced science and technology to propose a system which is more suitable for the present large-scale, field water quality monitoring.

The system is based on wireless sensor network technology and GPRS technology to monitor the water quality parameters of monitored area in real-time. Once the level of pollution in water of the monitored area, the pollution information can timely feedback to the total monitoring center [1]. It is convenient for management of the supervision department, at the same time it can help enterprises to monitor as well as determine if the factory discharge wastewater exceeds the standard or not, so as to timely adjust drainage condition, reduce water pollution, and avoid more serious environmental problems. The monitored water quality data can be used for historical query, so as to help the government and the relevant environmental protection departments to grasp the pollution characteristics of rivers, and further to estimate the river pollution, and take appropriate environmental protection measures in time [2-4]. The system can also connect to the Internet, so that the monitored water quality data can be brought into the public, help the public have a better understanding of the pollution of rivers in China, to inform those who lack environmental protection awareness.

The subject of this paper involves many areas of knowledge related to data acquisition technology, wireless sensor network technology, remote monitoring technology, automatic control and database management and other technical fields. The study of the entire water quality monitoring system plays an important guiding role in engaging subsequent related projects.

\section{Overall design of water quality monitoring system}

\subsection{ZigBee Topological structure}

The ZigBee network supports three types of network topology that contain master and slave devices: star network, cluster-tree network, and mesh network.

Star Network: The star network is composed of a network coordinator as a controller and a plurality of terminal nodes as slave devices. The center of the network reveals a diffusion type, with the network coordinator as the FFD. The terminal equipment is distributed around the network coordinator, and it can be FFD and can also be RFD. The network coordinator is responsible for the establishment and maintenance of the network. The terminal device communicates directly with the network coordinator, and the direct communication between each terminal device is not available, with all the information being forwarded through the network coordinator [5-6]. The structure of star network is simple, which is the most common network structure, has poor flexibility, usually using a small number of nodes covering a small area. The star network topology is shown in Figure 1.

Cluster-tree Network: A cluster-tree network consists of a network coordinator of a fully functional device and a number of star networks, including network coordinators, routers, and end devices. The network coordinator is also responsible for the establishment and maintenance of the network, with the router completing data rout- 
ing functions. The terminal devices still cannot directly communicate with each other, and the terminal device information is generally transmitted through the router to reach the network coordinator [7]. The cluster-tree network structure is relatively more complex than the star network. Having a relatively larger coverage area than the star network, the network scalability is very good, but the information transmission delay will increase, and in the case of a routing node failure a transmission path paralysis associated with the routing node wil result.

Mesh network: The mesh network consists of a network coordinator and a number of routers, and a part of the router then connects to the terminal equipment. The devices in the network are fully peer-to-peer [8]. Each device can communicate with other devices within the wireless communication range. Data forwarding generally takes the form of multi-hop. Each device has a forwarding function. The mesh network topology is shown in Figure 2.
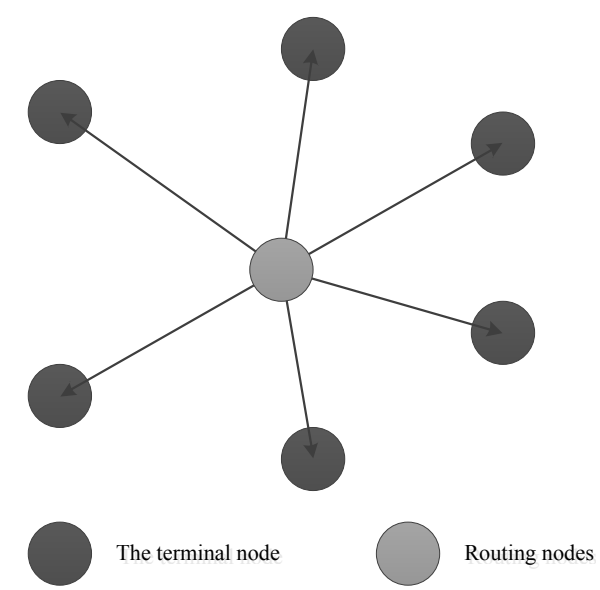

Fig. 1. Star network

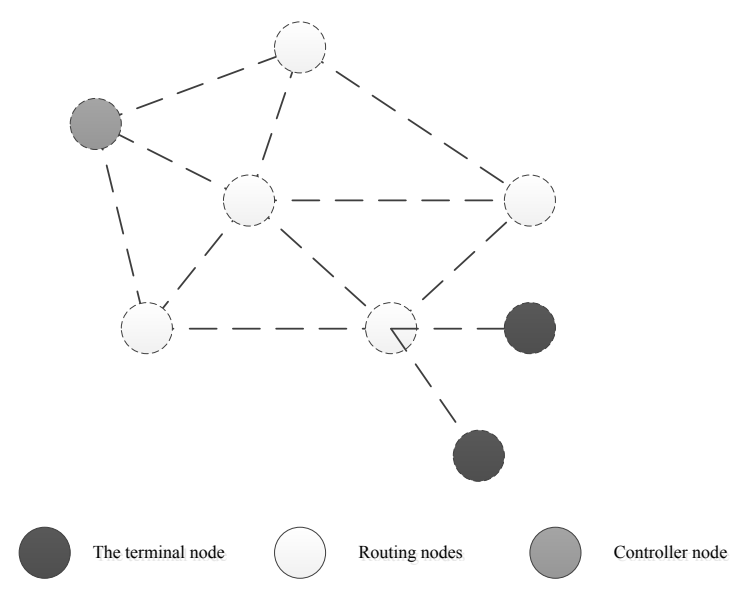

Fig. 2. Mesh network 


\subsection{Overall structure design of water quality monitoring system}

The monitoring of water quality has many characteristics, such as a large number of monitoring points in the region, long monitoring time and a complicated monitoring situation. Aiming to overcome the shortcomings of traditional water quality monitoring, such as long monitoring time and backward monitoring methods, a water quality monitoring system of a wireless sensor network based on ZigBee is designed. The water quality monitoring system consists of three parts: data acquisition, data remote transmission, and upper monitoring center. Figure 3 shows the structure of the water quality monitoring system [9].

\subsection{Monitoring node design}

The sensor node is the source of system data, and it is mainly composed of the sensor acquisition part, signal conditioning circuit, wireless communication part and power supply. The sensor node collects water quality parameters, and transmits its data to the host computer [10-12]. The sensor acquisition part is responsible for the perception of objects within the monitored area and data collection. The signal conditioning module is responsible for data conversion of the information collected by the sensor, and the wireless communication module can realize the wireless communication between the node and other sensor nodes, including information exchange and wireless reception and transmission of data. The power supply provides energy for the normal operation of sensor nodes. Figure 4 shows the structure of the system sensor nodes.

\section{Software design of water quality monitoring system}

\subsection{ZigBee module design}

The core part of the ZigBee module is comprised of a microprocessor and radio frequency, thus it is necessary to consider which wireless chips are suitable for the two parts. Under the circumstances that the basic design requirements have been met, the microprocessor and the radio frequency module also requires consideration of the following aspects:

1. High integration: Microprocessors require a high degree of integration, therefore the general choice is system-on-chip;

2. Low cost: In this system, a large number of sensors will be used. In order to reduce the total cost, the design of the sensors will be as simple and practical as possible;

3. Low power consumption: Considering that the system environment is in the field, the power supply cannot be adopted, so the system requires low power consumption. In order to reduce energy consumption, the system will also support a sleep mode. 


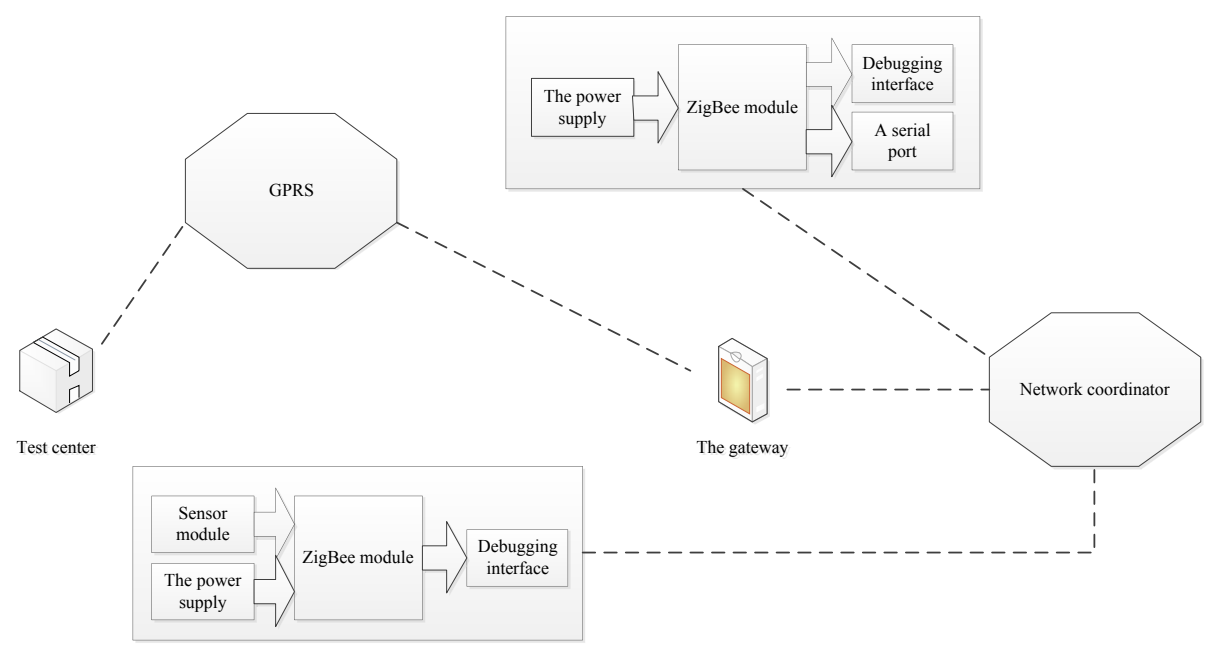

Fig. 3. Water quality monitoring system structure

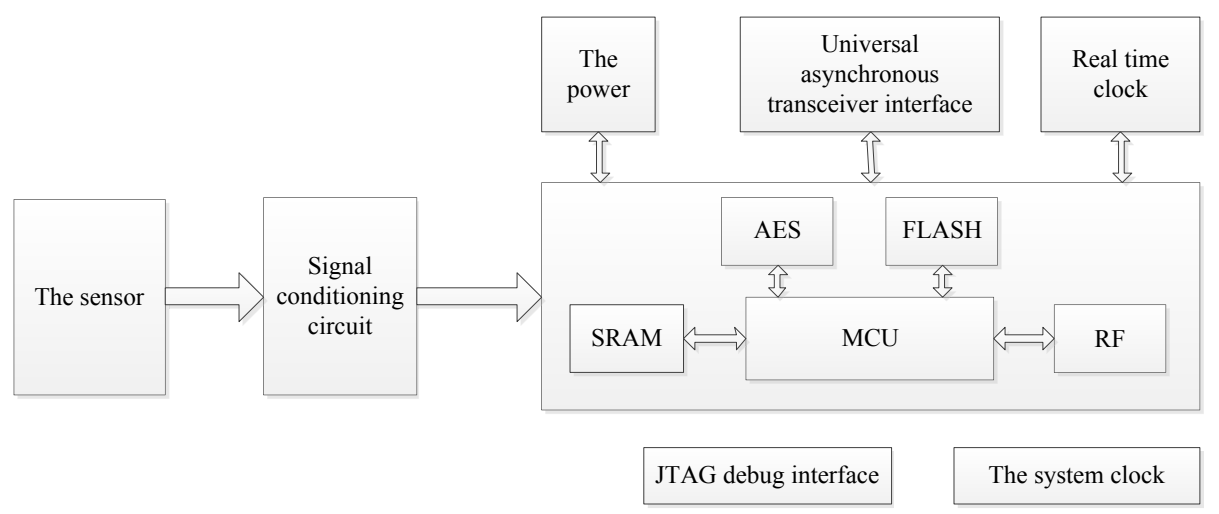

Fig. 4. Sensor node structure

For the RF module, good transmission performance is the criteria for choosing chips. Data transmission rate, distance and bit error rate directly affect the realization of system functions. Regarding anti-interference capability, wireless transmission is most prone to signal interference, which will result in data inaccuracy or distortion. Therefore, chips with high anti-interference capability should be chosen.

This system adopts the CC2430-F128 processor as the core, which supports 2.4GHz IEEE 802.15.4 ZigBee protocol. There are three versions according to different ROM sizes. CC2430-F32 / 64/128 correspond to the $32 \mathrm{~K} / 64 \mathrm{~K} / 128 \mathrm{~K}$ built-in flash memory, respectively. Its internal integrated enhanced industry standard 8051 core, radio frequency transceiver, $128 \mathrm{~K}$ built-in flash memory and $8 \mathrm{~K}$ static random access memory and other high-performance modules, in addition to the chip, has ZigBee protocol stack, low power consumption and other functions. These characteristics give the chip certain advantages in the formation of ZigBee nodes. On this basis 
CC2430 also has high sensitivity and immunity, with a built-in temperature sensor, battery monitor, supports a variety of communication protocols, and has supports for hardware debugging and other functions. Based on the above characteristics, the chip is able to meet the system design requirements, saves resources, and simplifies the overall design of the program.

\subsection{Minimum system design}

The minimum system includes processor, crystal oscillator clock, reset, antenna, power interface, JTAG and other parts.

Processor: In the processor, the different capacitance value of the decoupling capacitors will be connected between 7,20,23,23,25,27,28,35,38,41,47 pin and the ground in between for the RF load work so as to ensure the best gain. The packaging is 0402 , NP0-type capacitor with $5 \%$ precision and voltage $50 \mathrm{~V}$.

Clock: The system uses two crystal oscillators; that is, $32 \mathrm{MHz}$ system main clock and $32768 \mathrm{~Hz}$ real-time clock, X1, X2 as shown in Figure 5. X1 is NX3225SA-type 4pin patch passive crystal oscillator. Only 1,3 pin is valid and connected to $27 \mathrm{pF}$ and $33 \mathrm{pF}$ capacitors to ensure a higher accuracy of the system clock. X2 is an ordinary 2pin crystal oscillator, with two pins connected to a $15 \mathrm{pF}$ capacitor.

Antenna: The system designs two kinds of antenna scheme, and the choice of the antenna is decide by the jumper line One is the external SMA interface monopole antenna, which performs well and has a larger volume suitable for long-distance communication, such as the ANT components shown in Figure 5. The other kind of antenna scheme is the inverted "F"-shaped PCB antenna which is in accordance with CC2430 manual requirements. The PCB_ANT components are shown in Figure 5.

Reset: The system has three reset sources: key force reset, power-on reset, and watchdog reset. After reset, the memory and program are restored to the initial values.

\subsection{Design of serial port communication circuit}

The system serial interface uses a MAX232 chip which can provide two serial interface connections to achieve level conversion and complete communication with the host computer. The serial communication circuit is shown in Figure 6.

The service center generates a large number of key chains for users, which are divided into $m$ groups. For convenience of description, the number of key chains in each group is assumed to be $m=2^{k}, k$ is an integer, and the key chains in the $m$ group are adopted to generate lower-level allocation sub-tree for the key header chain according to the method in the previous section. Then, the upper-layer root allocation tree is constructed by the hash value of the root information of the $m$ sub-trees as the leaf node, as shown in Figure 6. 


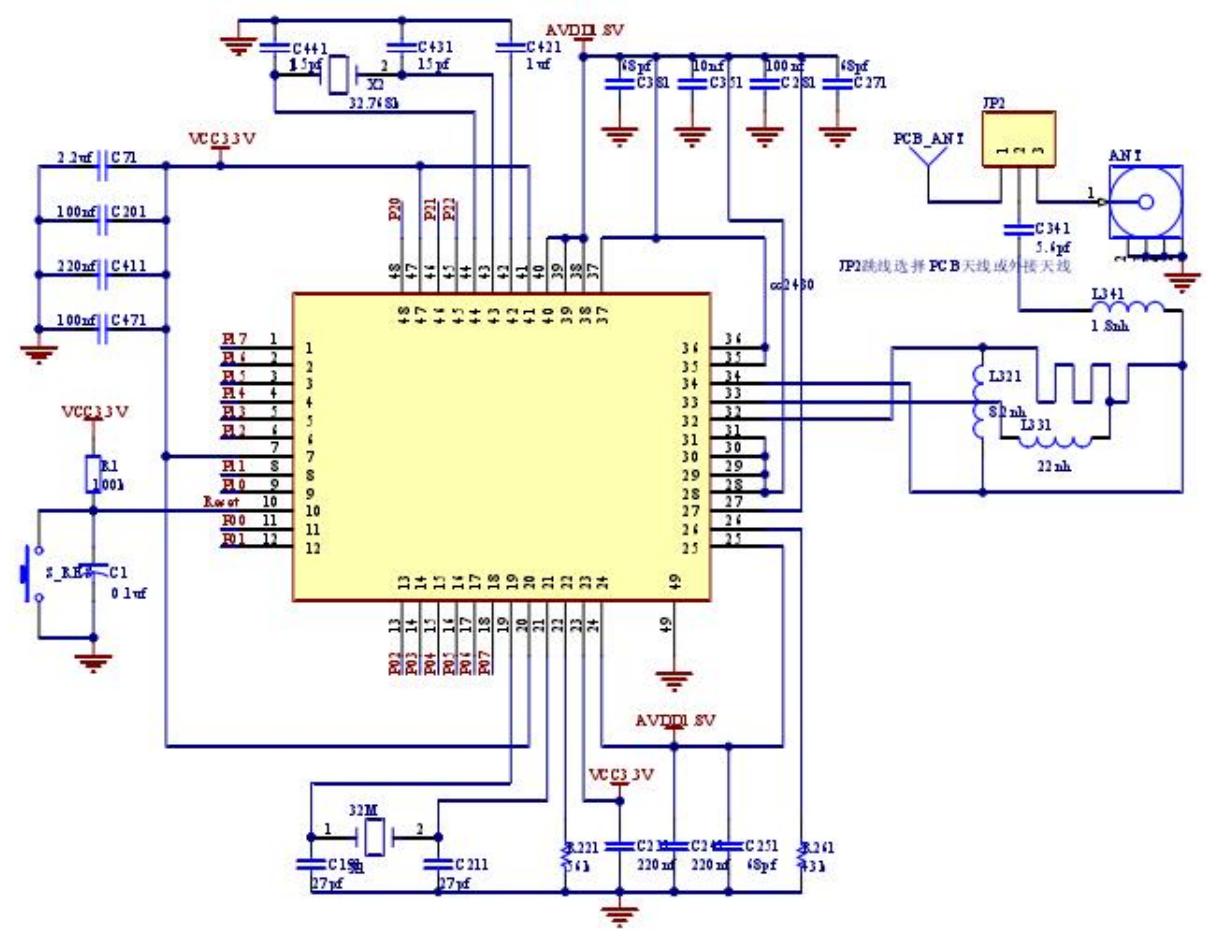

Fig. 5. Minimal system

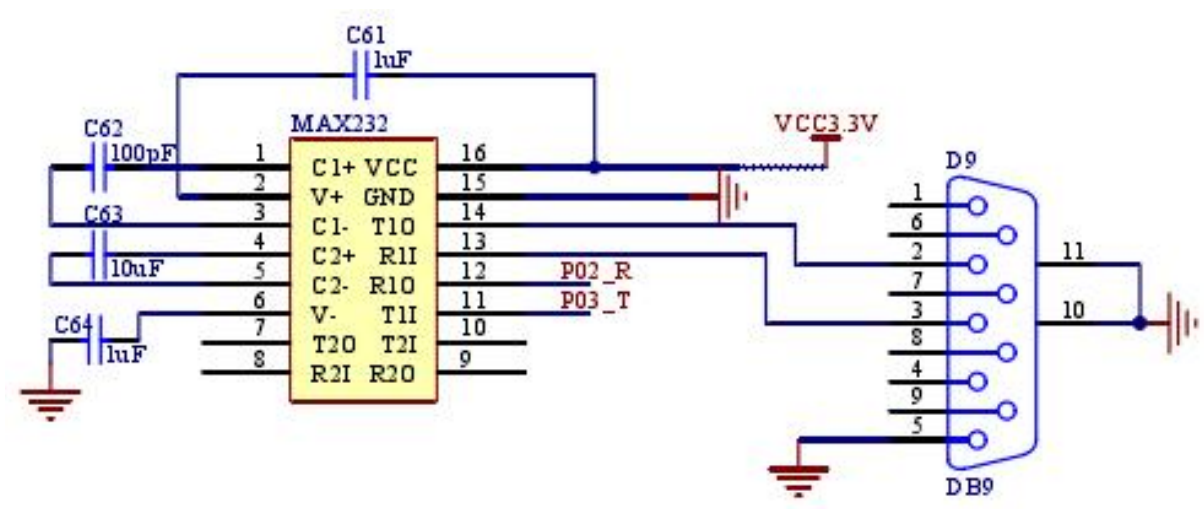

Fig. 6. Serial port communication circuit 


\section{Software design of water quality monitoring system}

\subsection{Z-Stack protocol stack}

Z-Stack Protocol stack is the protocols defined by IT companies in the communication system which stipulates how hardware and software work together at different layers. In network communication, the data must be packaged before communication, and after packaging, the entities from different layers can communicate with the peer entity. If the data packages delivered by the sender user want to pass through the protocol layer, it is according to the order from the upper layer to the lower layer, and each layer adds its own information according to the specified format. After transmission, the data package will finally arrive at the physical layer and transmit to each other between the physical connections. While the receiver's data package must pass through the protocol layer from the lower to the upper layer, each layer extracts the information which must be processed in this layer. The end user obtains the data information of the data package through the applications, and then further processing is conducted, as shown in the flow chart in Figure 7.

\subsection{Service primitives}

The IEEE 802.15.4 and Zig Bee standards use the notion of primitives to describe a layer providing services to higher layers. The adjacent layers call function or transfer information through the service primitive so as to realize communication management of protocol layer. There are four types of service primitives: Request, Indication, Response, and Confirm.

1. Request: It is transmitted from a lower layer to an upper layer, used for request of service;

2. Indication: It is transmitted from an upper layer to a lower layer, used to indicate that in an event the upper layer is of important significance for the lower layer. This event may be logically related to a remote service request or may be caused by an internal event;

3. Response: It is transmitted from a lower layer to an upper level, in response to service procedures call by the previous indication;

4. Confirm: It is transmitted from an upper layer to a lower layer, used for confirmation of the result of the previous one or more related service requests. 


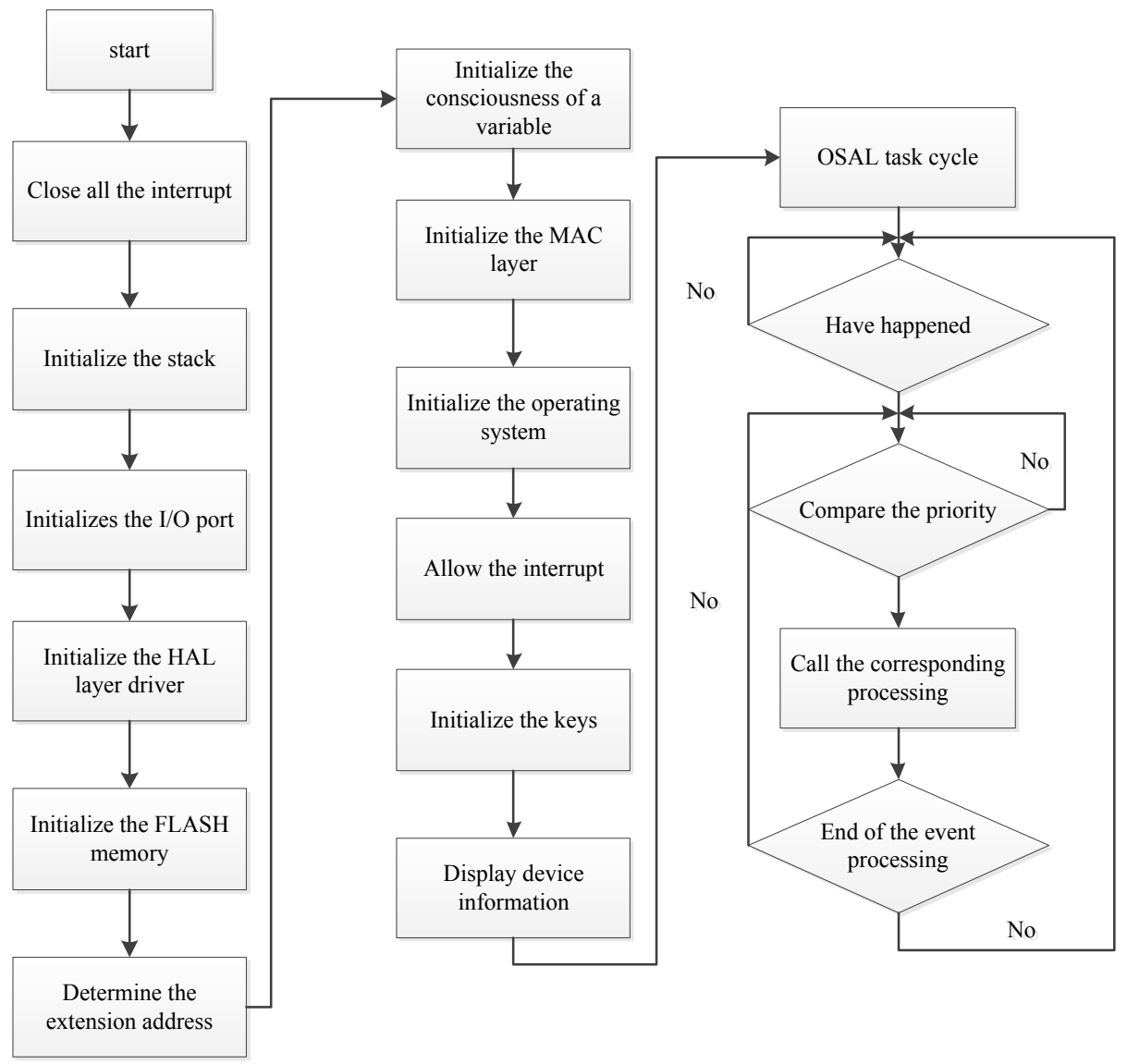

Fig. 7. Z - Stack operation flow chart

\section{$5 \quad$ System test results and analysis}

\subsection{Sensor node data acquisition}

The sensor transmits the data to the sensor node through the conditioning circuit, and the sensor node realizes temperature collection through the temperature sensor. In order to verify the accuracy of the measured temperature and $\mathrm{PH}$ value, this study selected three different temperatures of water and three common solutions for measurement and comparison. Using the laboratory thermometer and temperature sensor to measure the three kinds of water with different temperatures, as shown in Table 1, the measurement results of the two methods are similar. With the presence of artificial measurement error, it can be considered that the measurement accuracy of the sensor is good. 
In this study, three kinds of solutions, namely mineral water, green tea and cola, were selected for sampling and to measure their corresponding PH values. The results were compared with those measured by the laboratory $\mathrm{PH}$ meter. It can be seen in Table 2 that the measured PH basically reflects the PH value of the corresponding solution, but the error is large, and the output display during the measurement is very unstable. This may be due to the following reasons. First, the PH electrode calibration may have an error and during the electrode calibration and measurement, the ambient temperature may change. Second is a discharge voltage imbalance. Third, during the design of the conditioning circuit, the data interference considerations are not comprehensive. The follow-up study will be based on corrections to the above reasons so as to improve the measurement accuracy of the $\mathrm{PH}$ value.

Table 1. Water temperature measurements value

\begin{tabular}{|l|l|l|l|}
\hline Water temperature meter measuring values & 15.2 & 24.1 & 42.3 \\
\hline Sensor measurements value & 15.4 & 25.3 & 42.6 \\
\hline
\end{tabular}

Table 2 Comparison of PH Value

\begin{tabular}{|l|c|c|c|}
\hline & Mineral water & Green tea & Cola \\
\hline Output voltage measurement & 1.23 & 1.23 & 0.52 \\
\hline PH measurement & 5.26 & 5.69 & 2.58 \\
\hline Standard PH & 6.52 & 6.25 & 3.39 \\
\hline
\end{tabular}

\section{$5.2 \quad$ Networking}

The main function of the network coordinator is to start the network, which is implemented by channel scanning, immediately starting a network when unused channels are found. The function of the router is to expand the ZigBee network through channel scanning, which when finds an existing activated channel then joins them, while at the same time allowing other devices to connect with the network. Additionally, the terminal devices attempt to join an existing network by sending a request to a network coordinator or router.

When the network coordinator establishes a new network, the network layer first requests the MAC layer to perform an energy scan and then performs an active scan on the responding channel. If the network layer cannot select a suitable channel, then the network setup will fail. If a suitable channel is selected and the PAN identifier PAN ID is determined, then the network management entity selects $0 \times 0000$ as the 16bit network address, which will result in networking success. The node then scans the channel to determine if there is a network coordinator and then issues an application, the network coordinator will respond to the node. When the node receives three responses, it will send 64-bit physical addresses to the network coordinator and apply to join the network. After receiving the application, the network coordinator assigns a 16-bit network address to the node, and the node successfully joins the network.

In general, the ZigBee network includes a network coordinator and a terminal node. When the network is large, there may be routing nodes. The node type is deter- 
mined by the network topology, indicating that the ZigBee network must be determined according to the actual situation. The network coordinator and sensor nodes are included in the network, and when the sensor node is a full-function device, it is basically the same as the routing node.

The programs are downloaded to three ZigBee modules for networking, and the network includes a network coordinator and two sensor nodes. The serial port network coordinator and PC were connected. In the experiment, firstly the power is turned on in the network coordinator. The network coordinator sends its network address to the PC through the serial port, and then power is turned on in the other two modules. If a network coordinator is powered, it will automatically establish a network, indicated with LED lights, and then the power supply is opened for the other two sensor nodes, which will automatically join the network, indicated by the corresponding LED lights.

\section{Conclusion}

A large number of references have been analyzed in this paper, supported by advanced technologies such as the wireless sensor network and ZigBee technology. On the basis of comprehensively discussing the various factors of the system, the system structure is constructed and the system development and debugging are completed. After testing the performance of the system hardware, through downloading the corresponding program of different functions, the joint debugging of system hardware and software has been realized, so that each node is able to complete its own functions. In this way, the system will be able to achieve collection and transmission of water quality parameters.

\section{$7 \quad$ References}

[1] Akkaya K., Younis M. (2005). A survey on routing protocols for wireless sensor networks. Ad Hoc Networks, 3(3), 325-349. https://doi.org/10.1016/j.adhoc.2003.09.010

[2] Van Dam T., Langendoen K. (2003). An adaptive energy-efficient MAC protocol for wireless sensor networks. International Conference on Embedded Networked Sensor Systems, 67, 23-27. https://doi.org/10.1145/958491.958512

[3] Karlof C., Wagner D. (2003). Secure routing in wireless sensor networks: attacks and countermeasures. IEEE International Workshop on Sensor Network Protocols and Applications, Proceedings of the First IEEE, 1, 113-127. https://doi.org/10.1109/SNPA.2003. 1203362

[4] Du W., Deng J., Han Y.S., Varshney P.K. (2005). A pairwise key pre-distribution scheme for wireless sensor networks. Acm Transactions on Information \& System Security, 8(2), 228-258. https://doi.org/10.1145/1065545.1065548

[5] Patwari N., Ash J.N., Kyperountas S., Hero A.O.I., Moses R.L., Correal N.S. (2005). Locating the nodes: cooperative localization in wireless sensor networks. IEEE Signal Processing Magazine, 22(4), 54-69. https://doi.org/10.1109/MSP.2005.1458287 
[6] Doherty L., Pister K.S.J., Ghaoui L.E. (2001). Convex position estimation in wireless sensor networks. INFOCOM 2001. Twentieth Joint Conference of the IEEE Computer and Communications Societies. Proceedings. IEEE, 3, 1655-1663.

[7] Bandyopadhyay S., Coyle E.J. (2003). An energy efficient hierarchical clustering algorithm for wireless sensor networks, 3(3), 1713-1723.

[8] Du W., Deng J., Han Y.S., Varshney P.K. (2003). A pairwise key pre-distribution scheme for wireless sensor networks. ACM Conference on Computer and Communications Security, 8, 228-258. https://doi.org/10.1145/948109.948118

[9] Abbasi A.A., Younis M. (2007). A survey on clustering algorithms for wireless sensor networks. Computer Communications, 30(14-15), 2826-2841. https://doi.org/10.1016/ j.comcom.2007.05.024

[10] Ganesan D., Govindan R., Shenker S., Estrin D. (2001). Highly-resilient, energy-efficient multipath routing in wireless sensor networks. Acm Sigmobile Mobile Computing \& Communications Review, 5(4), 72-85. https://doi.org/10.1145/509506.509514

[11] Boyinbode O., Le H., Mbogho A., Takizawa M. (2010). A Survey on Clustering Algorithms for Wireless Sensor Networks. International Conference on Network-Based Information Systems, 30, 358-364. https://doi.org/10.1109/nbis.2010.59

[12] Krishnamachari B., Estrin D., Wicker S. (2002). The impact of data aggregation in wireless sensor networks. International Conference on Distributed Computing Systems Workshops, 5, 575-578. https://doi.org/10.1109/ICDCSW.2002.1030829

\section{Authors}

Minhai Zhang is a lecturer with Hunan Vocational College of Railway Technology, Zhuzhou 412006, China. He is mainly engaged in the study of Electronic communication (lishijun040482@qq.com)

Shuangxiang She is a master of Powerchina Zhongnan Engineering Co Ltd., Changsha 410014, China. He is mainly engaged in communication technology research (15116380139@126.com).

Article submitted 04 February 2017. Published as resubmitted by the authors 10 March 2017. 